Sādhanā Vol. 40, Part 4, June 2015, pp. 1077-1089. (C) Indian Academy of Sciences

\title{
Profit allocation among rational players in a cooperative game under uncertainty
}

\author{
SIBASIS BANDYOPADHYAY ${ }^{1}$, SWAPAN RAHA ${ }^{1}$ and \\ PRASUN KUMAR NAYAK ${ }^{2, *}$
}

${ }^{1}$ Department of Mathematics, Visva Bharati, Santiniketan- 731 235, India
${ }^{2}$ Bankura Christian College, Bankura- 722 101, India
e-mail: sibasisbanerjee@ rediffmail.com; swapan.raha@ visva-bharati.ac.in;
nayak_prasun@ rediffmail.com

MS received 17 May 2014; revised 19 September 2014; accepted 21 November 2014

\begin{abstract}
In this paper, a cooperative game has been considered, where there is a coalition between rational players in an uncertain environment. The uncertainty occurs in terms of the quality of the raw material. The pay-offs of the players are influenced by the degree of satisfaction. Pay-offs are considered as interval numbers. A multisection technique has been applied to obtain a rational solution. In the process, it has been found that the degree of satisfaction of the rational players converges to its optimum value with the convergence of the iterations. A comparative study has been made with methods existing in the literature.
\end{abstract}

Keywords. Cooperative game; fuzzy numbers; multisection technique.

\section{Introduction}

In game theory, an individual's self-interest is a fundamental aspect. In real world, there are numerous instances where an individual participates in coalitions e.g., family, firms, market places and political jurisdiction. There is a wide range of commodity distribution and quasiconcave preferences in a modern highly polished socio-economic framework motivating to remain in the position to bargain as a part of a coalition. This situation gives birth to the idea of co-operative game with coalition of individuals. In general, a game is defined as a particular course of action between two or more players under a set of rules. In modern society any conflict of interest situation that arises in business, politics, etc. can be handled by game theory. But, imprecision of information is a fundamental problem in industrial problems. This imprecision is modelled by fuzzy set and the corresponding game is then called a fuzzy game. The reward that a player gets at the end of the game is called pay-off. In a crisp sense, co-operative game can be considered as a function $v: 2^{n} \rightarrow R^{+} \bigcup\{0\}$ such that $v(\phi)=0$ where $N=\{1,2, \ldots n\}$ denotes the set of players participating in the coalition and for each coalition $\widetilde{s} \in 2^{n}$, the real number $v(\widetilde{s})$ is considered to be worth the participation $\widetilde{s}$. This participation may be total or nil

*For correspondence 
in a crisp sense or partial in a fuzzy sense. A significant contribution has been made in fuzzy cooperative game by researchers (Xiaodong et al 2013; Aubin 1982; Butnariu 1980; Branzei et al 2004, 2005). They gave a detailed explanation on the term 'fuzzification' with respect to individual participation in the coalition. On the other hand, Mares \& Vlach (2001, 2006), Romero Cortes \& Sheremotov (2002) considered uncertainty in the value associated with the characteristic function of a game. They worked on a crisp or deterministic coalition although the outcome was fuzzy quantities. Borkotokey (2008) explained a cooperative game where both the coalition and the characteristic function were fuzzy. There was however a need to find ways to distribute the profit or worth of a fuzzy or crisp coalition among the individuals participating in the coalition. Shapley (1953) introduced some concepts to deal with such a situation. In fact, further research was carried out in two ways on dynamic coalition formation. Shapely values, core and minimum norm solution in Aubin (1982) are static in nature. Research carried out by Dieckmann (2002); Ray \& Vohra (1997), (1999), (2001) considered solutions under dynamic coalition. But these solution methods could not provide a rational allocation of profit or worth that also considers the level of satisfaction of the participating players. Borkotokey \& Rupok (2012) offered in their model a dynamic approach to arrive at a suitable solution of a cooperative fuzzy game. They proposed an efficient allocation of profit among rational players such that they have almost equal satisfaction. They also introduced the concept of penalty to maintain a fair bargaining process. But this allocation of profit was not that rational when compared to supply of products. This shortcoming motivated the search for a new technique that was attempted in this paper. We have considered that in the coalition, made in an fuzzy environment modelled by intuitionistic number where not only the amount of participation, the quality assessment of the participation is also taken into account. Here we take an intuitionistic fuzzy number to model the technique because here the contribution is also taken with respect to acceptance and rejection degree of a decision maker to consider whether the product supplied is good or not. This intuitionistic fuzzy coalition corresponds to a crisp allocation of profit which are considered as interval numbers. A single satisfaction function is defined for all players in such a manner that there is no need for separate bargaining. Then a multi-section technique has been applied to each interval to get the ultimate solution. The multi-section technique is based on the idea of bisection (Sakawa 1983) and it is used for searching a solution by repeatedly dividing the range of the variable into multiple parts i.e. multiple bisection is done at single iteration cycle. Here, the allocation has been made on the basis of coalition in such a rational way that there is no need to explain the penalty separately.

(i) A multisection technique (Nayak et al 2014) has been applied to our proposed model to get the profit allocation with respect to the supply of best quality of products which gives a more rational result compared to Borkotokey \& Rupok (2012) and at the same time, inspires the fair participation of players with substantial honesty.

(ii) The satisfaction function has been considered as an exponential function which increases the rate of convergence as compared to Borkotokey \& Rupok (2012).

(iii) The interval range of profit allocation has been made under the influence of quality perspective of participation so that it carries out the bargaining process with the rationalization of satisfaction. In Borkotokey \& Rupok (2012) there is no scope for quality assessment of products.

Here, it is not necessary to describe the bargaining process separately because the multisection technique justifies automatically the degree of satisfaction with the iterations when applied over the interval allocation of profit. In Section 2, some basic definitions, notations and 


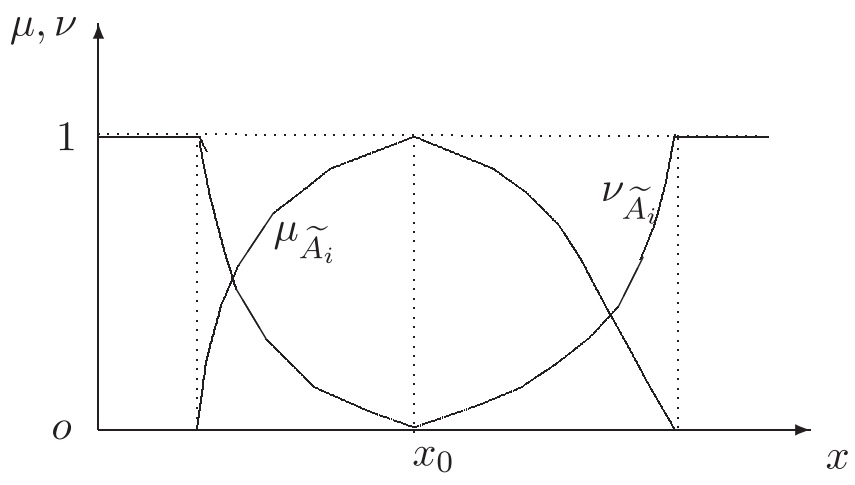

Figure 1. Membership and non-membership functions of $\widetilde{A}_{i}{ }^{i}$.

preliminaries have been described. In Section 3, we have described our model. In Section 4, an exact solution searching protocol has been proposed. In Section 5, an example is provided in support of the performance of the proposed method and along with explanations, a comparative study related to algorithm and results of the numerical example.

\section{Preliminaries}

In this section, we discuss the concept of an intuitionistic fuzzy coalition in a cooperative game. An intuitionistic fuzzy $\operatorname{set}(\widetilde{A})$ (Seikh et al 2013) is characterized by an ordered triplet as

$$
\widetilde{A}=\left\{\left(x, \mu_{\widetilde{A}}(x), v_{\widetilde{A}}(x)\right) \mid x \in X\right\},
$$

where $X$ is the universe of discourse, $\mu_{\widetilde{A}}(x): X \rightarrow[0,1]$ is a membership function which measures the degree of belongingness of an element $x$ to the subset $\widetilde{A}$ of $X, v_{\widetilde{A}}(x): X \rightarrow[0,1]$ is a non-membership function which measures the non-belongingness of an element $\mathrm{x}$ to the subset $A$ of $X$. Here, for membership function $\mu_{\widetilde{A}}(x)$ and non-membership functions $v_{\widetilde{A}}(x)$ the condition $0 \leq \mu_{\widetilde{A}}(x)+v_{\widetilde{A}}(x) \leq 1$ exists.

An intuitionistic fuzzy number $\widetilde{A}^{i}$ is defined (figure 1 ) as a convex normalized fuzzy set $\widetilde{A}^{i}$ over the real line $\mathfrak{R}$ for membership function and concave normalized fuzzy set over real line $\Re$ for non-membership functions satisfying the following conditions:

(i) $\exists$ exactly one point $x_{0}$ such that for $x_{0} \in \Re, \mu_{\widetilde{A}^{i}}\left(x_{0}\right)=1$ and $v_{\widetilde{A}^{i}}\left(x_{0}\right)=0$. Here $x_{0}$ is called mean value of $\tilde{A}^{i}$.

(ii) $\mu_{\widetilde{A}^{i}}(x)$ and $\nu_{\widetilde{A}^{i}}(x)$ are piecewise continuous.

Here, we consider a special class of fuzzy game proposed by Azrieli \& Lehrer (2007) which gives a more generalized class of fuzzy games compared to existing classes. In our method, we consider a population of size $n$ consisting of a continuum of each type of agents represented by a non-negative vector $Q=\left(Q_{1}, Q_{2}, \ldots Q_{n}\right)$ where $Q_{i} \geq 0$ is the size of the type $i(i=1,2, \ldots n)$ agents (Borkotokey \& Rupok 2012). We can formalize it as

$$
F(Q)=\left\{s \in \Re^{n}: 0 \leq s \leq Q\right\} .
$$

Here, we interpret $Q$ as the 'Grand coalition' for every possible coalition $s \in F(Q)$. 
Let a real number $v(s)$ represent the total profit resulting from the intuitionistic fuzzy coalition $s=\left(c_{1}, c_{2}, \ldots c_{n}\right)$ between the agents of the population and $c_{i}$ represent the amount with which an agent of type $i(i=1,2, \ldots, n)$ participate in the coalition. Thus for $Q \geq 0: Q \in \Re^{n}$, we mathematically define a cooperative game $(Q, v)$ as

(i) $Q \in \Re^{n}$ and $Q \geq 0$.

(ii) $v: F(Q) \rightarrow \mathfrak{R}^{+} \bigcup 0$ is bounded and satisfies $v(0)=0$.

Here, in a coalition we consider that each agent can invest any fraction of his resources $c_{i} \leq Q_{i}(i=1,2, \ldots, n)$ in Aubin's (Aubin 1982) sense. In fact, we work not with the amount $c_{i}(i=1,2, \ldots, n)$ that an agent invests but with the fraction of the original resources that he invests. We emphasis also on the quality of his product that he invests in the coalition. We often see in a cooperative business that if an agent participates with low quality product then the total profit decreases. So that agent should take responsibility for such a situation. For this reason, we make a quality assessment of each contribution and consider an intuitionistic fuzzy number $\widetilde{A}_{i}=$ $\left\{\left(Q_{i}, \mu_{\widetilde{A}_{i}}\left(Q_{i}\right), v_{\widetilde{A}_{i}}\left(Q_{i}\right)\right) \mid Q_{i} \in Q\right\}(i=1,2, \ldots, n)$ instead of $c_{i}$ where $\mu_{\widetilde{A}_{i}}\left(Q_{i}\right)$ represents the best quality products that he delivers in accordance with the standard market measurement. $v_{A_{i}}\left(Q_{i}\right)$ represents the lower quality product that he invests in the coalition and $\pi_{i}\left(Q_{i}\right)=$ $1-\left(\mu_{\widetilde{A}_{i}}\left(Q_{i}\right)+v_{\widetilde{A}_{i}}\left(Q_{i}\right)\right)$ the uncertainty in quality measurement. So, we give an alternative definition of a cooperative game in intuitionistic fuzzy environment as a pair $(\widetilde{Q, v})$ such that

(i) $Q \in \Re$ and $Q \geq 0$.

(ii) $v: \widetilde{F}(Q) \rightarrow \mathfrak{R}^{+} \cup 0$ is bounded and satisfies $v(0)=0$.

where $\widetilde{F}(Q)=\left\{\widetilde{s}: 0 \leq \widetilde{s} \leq \widetilde{A_{i}}\right\}$ and $\widetilde{s}=\left(\widetilde{A_{1}}, \widetilde{A_{2}}, \ldots, \widetilde{A_{n}}\right)$ represents the "possible intuitionistic coalition". $\widetilde{s}_{i}=\left(0,0, \ldots, \widetilde{A}_{i}, \ldots, 0\right) \in \widetilde{F}(Q)$ represents contribution of a particular agent $i(i=1,2, \ldots, n)$ in the intuitionistic fuzzy cooperative game. The set $\left\{i \in N \mid \widetilde{A}_{i} \geq 0\right\}$ supports $\widetilde{s}$. For better interpretation of our theory we propose some more definitions.

Definition 2.1 A cooperative fuzzy game in intuitionistic fuzzy environment is said to be super additive if $v(\widetilde{s} \bigvee \widetilde{u}) \geq v(\widetilde{s})+v(\widetilde{u})$ for every $\widetilde{s}, \widetilde{u} \in \widetilde{F}: \widetilde{s} \wedge \widetilde{u}=0 . G_{\widetilde{F}}(Q)$ represents the class of superadditive cooperative intuitionistic fuzzy game with respect to grand intuitionistic coalition $Q$.

Definition 2.2 An allocation is a vector of pay-offs $x=\left(x_{1}, x_{2}, \ldots, x_{n}\right) \in \Re^{n}$. The allocation $x$ is efficient for coalition $\widetilde{s}$ if $\sum_{i=1}^{n} x_{i}=v(\widetilde{s})$.

Definition 2.3 Let $\widetilde{s}_{i}=\left(0,0, \ldots, \widetilde{A_{i}}, \ldots, 0\right) \in \widetilde{F}(Q)$. Then the minimum deal index of a cooperative intuitionistic fuzzy game $v \in G_{F}(Q)$ with respect to an intuitionistic fuzzy coalition $\widetilde{s}$ is defined as a vector $x(i, \widetilde{s}) \in \Re^{n}$ such that

$$
x(i, \widetilde{s})= \begin{cases}v\left(\widetilde{s_{i}}\right)+\left(\widetilde{A_{i}} / \sum_{i} \widetilde{A}_{i}\right)\left[v(\widetilde{s})-\sum_{i=1}^{n} v\left(\widetilde{s_{i}}\right)\right] & \text { if } i \in \text { Supp } \widetilde{s} \\ 0 & \text { otherwise }\end{cases}
$$

It can be noted that minimum deal index is an efficient allocation. For further development of the theory we need to explain an interval number as the algorithm applied here is based on the interval number. 
Definition 2.4 An interval, Moore (1979), is defined as

$$
\bar{a}=\left[a_{L}, a_{R}\right]=\left\{x: a_{L} \leq x \leq a_{R}, a_{L} \in \Re, a_{R} \in \Re\right\},
$$

where $a_{L}$ and $a_{R}$ can be explained as lower and upper limits of the interval $\bar{a}$ respectively.If $a_{L}=a_{R}$ then $\bar{a}=\left[a_{L}, a_{R}\right]$ is reduced to a real number $a$, where $a=a_{L}=a_{R}$. An interval $\bar{a}$ can alternatively be expressed in mean-width or center-radius form as $\bar{a}=\langle m(\bar{a}), w(\bar{a})\rangle$, where $m(\bar{a})=1 / 2\left(a_{L}+a_{R}\right)$ and $w(\bar{a})=1 / 2\left(a_{R}-a_{L}\right)$ are respectively the mid-point and half-width of the interval $\bar{a}$. The set of all interval numbers in $\Re$ is denoted by $I(\Re)$ where $\Re$ is the set of all real numbers.

\section{Mathematical model}

Let us consider a cooperative game $(\widetilde{Q, v})$ in intuitionistic fuzzy environment where $\widetilde{s}=$ $\left(\widetilde{A_{1}}, \widetilde{A_{2}}, \ldots, \widetilde{A_{n}}\right) \in \widetilde{F}(Q)$ with $\widetilde{s_{i}}=\left(0,0, \ldots, \widetilde{A_{i}}, \ldots, 0\right)$. We then, define a satisfaction function $S_{(\widetilde{s}, v)}^{i}: \Re \rightarrow[0,1]$ of the player $i$ over an intuitionistic fuzzy coalition $\widetilde{s} \in G_{\widetilde{F}}(Q)$ as

$$
S_{(\widetilde{s}, v)}^{i}=e^{-\frac{\left|v\left(\widetilde{s_{i}}\right)-\bar{v}\left(\widetilde{s_{i}}\right)\right|}{\left|v(\widetilde{s})\left(\mu_{\widetilde{A_{i}}}-\bar{\mu} \widetilde{A_{i}}\right)\right|}}=e^{-K_{i}}
$$

where $K_{i}=\frac{\left|v\left(\widetilde{s_{i}}\right)-\bar{v}\left(\widetilde{s_{i}}\right)\right|}{\left|v(\widetilde{s})\left(\mu_{\widetilde{A_{i}}}-\bar{\mu} \widetilde{A_{i}}\right)\right|}$ and $\bar{\mu} \widetilde{A_{i}}=1 / n \sum_{i=1}^{n} \mu_{\widetilde{A_{i}}}$ are mean of all $\mu_{\widetilde{A_{i}}}$ over the agent $i(i=1,2, \ldots, n)$ and $\bar{v}\left(\tilde{s}_{i}\right)$ are the mean of all $\tilde{s}_{i}$ over the agent $i(i=1,2, \ldots, n)$. The satisfaction $S_{(\widetilde{s}, v)}^{i}$ represents the satisfaction of a particular agent $i$ for a particular deal at a particular level. The reason behind taking such function as satisfaction function is that the exponential part actually represents the ratio of profit distribution and contribution of good products that results in rationalization of profit distribution in comparison with contribution of good quality product in subsequent iterations. Then we see that $S^{i} \rightarrow 0$ as $K_{i} \rightarrow \infty$ i.e., when the difference of allocation of profit and mean allocation is very large compared to the difference of supply of the best quality product and average supply of best quality product then the satisfaction of the agent $i$ is small. Again, when $K_{i} \rightarrow 0$ then $S_{\widetilde{s}, v)}^{i} \rightarrow 1$ i.e., when difference of profit of allocation and mean profit allocation is very small compared to the difference of supply of best quality product and average supply of best quality product then, the satisfaction of the agent $i$ is very close to 1 . This implies that if the allocation of the profit is rational compared to supply of its best quality product then its degree of satisfaction is very close to 1 . Here, the satisfaction of one agent does not depend on the satisfaction of another agent. It can be proved by the following theorem:

Theorem 3.1 The set of satisfaction functions $\left\{S_{(\widetilde{s}, v)}^{i}: i=1,2, \ldots, n\right\}$ is linearly independent.

Proof. Let $\alpha_{1}, \alpha_{2}, \ldots, \alpha_{n} \in \Re$ are arbitrary constants such that

$$
\sum_{i=1}^{n} \alpha_{i} S_{(\widetilde{s}, v)}^{i}=0 \quad \Rightarrow \sum_{i=1}^{n} \alpha_{i} e^{-K_{i}}=0
$$


Now this is true for any set of values of $\left\{K_{1}, K_{2}, \ldots, K_{n}\right\}$. Let $K_{i}=0$ and $K_{j} \rightarrow \infty$ for $j=1,2, \ldots, n$ such that $j \neq i$.Then

$$
\begin{aligned}
& \alpha_{1} .0+\alpha_{2} .0+\cdots+\alpha_{i} \cdot 1+\cdots+\alpha_{n} \cdot 0=0 \\
& \quad \Rightarrow \alpha_{i}=0
\end{aligned}
$$

Similarly we can prove that $\alpha_{j}=0$ for $j=1,2, \ldots, n$ and $j \neq i$.

Hence, $\left\{S_{\widetilde{s}, v)}^{i}: i=1,2, \ldots, n\right\}$ is linearly independent.

The question that arises is what is the actual domain of $\left\{S_{(\widetilde{s}, v)}^{i}\right\}$ for a particular agent $i$. To solve this question we further need to develop the theory by some definitions.

Definition 3.1 The $+_{1}$ quality index $p_{\widetilde{A_{i}}}: \widetilde{A_{i}} \rightarrow \Re$ of a particular agent $i$ is defined as $p_{\widetilde{A_{i}}}=$ $\left(\mu \widetilde{A_{i}}-\mu\right) / \mu_{\widetilde{A_{i}}}$ where $\mu=\min _{i} \mu_{\widetilde{A_{i}}}$ and $\mu \widetilde{A_{i}}$ represents the percentage of best quality of product it supplies with respect to its total source of product.

Definition 3.2 The $+_{2}$ quality index $q_{\widetilde{A_{i}}}: \widetilde{A_{i}} \rightarrow \Re$ of a particular agent $i$ is defined as $q \widetilde{A_{i}}=$ $\left(v-v \widetilde{A_{i}}\right) / v$ where $v=\max _{i} v{\widetilde{A_{i}}}$ and $v_{A_{i}}$ represents the percentage of low quality of product it supplies with respect to its total source of product.

Definition 3.3 The +3 quality index $r_{\widetilde{A_{i}}}: \widetilde{A_{i}} \rightarrow \Re$ of a particular agent $i$ is defined as $r_{\widetilde{A_{i}}}=$ $\left(\pi-\pi \widetilde{A_{i}}\right) / \pi$ where $\pi=\max _{i} \pi \widetilde{A_{i}}$ and $\pi \widetilde{A_{i}}$ represents the percentage of uncertain quality of product it supplies with respect to its total source of product.

Definition 3.4 The quality index $T_{\widetilde{A_{i}}}: \widetilde{A}_{i} \rightarrow \Re$ of a particular agent $i$ is defined as $T_{\widetilde{A_{i}}}=\left(p_{\widetilde{A_{i}}}+q_{\widetilde{A_{i}}}+r_{\widetilde{A_{i}}}\right) / 3$ where $T_{\widetilde{A_{i}}}$ represents the percentage of average quality of product it supplies with respect to its total source of product.

Here, we can observe that these quality indices actually represent the fact that if an agent supplies the better quality of a product then he will have a better quality index. We propose to show that the better quality index increases its profit allocation and for that purpose we will consider an interval around each deal index number $x(i, \widetilde{s})$ which gives the range of profit allocation for each agent $i$.

Let $x(i, \widetilde{s})$ be the minimum deal index of an agent $i$. Then the interval allocation of the profit of that particular agent is given by

$$
\begin{aligned}
I_{\widetilde{s}, v)}^{i} & =\left[x(i, \widetilde{s}), x(i, \widetilde{s})+x(i, \widetilde{s})\left(T_{\widetilde{A_{i}}}-\bar{T}_{\widetilde{A}_{i}}\right)\right] \text { if } T_{\widetilde{A_{i}}} \geq \bar{T}{\widetilde{A_{i}}} \\
& =\left[x(i, \widetilde{s})-x(i, \widetilde{s})\left(\bar{T}_{\widetilde{A}_{i}}-T_{\widetilde{A_{i}}}\right), x(i, \widetilde{s})\right] \text { if } T_{\widetilde{A_{i}}}<\bar{T}{\widetilde{A_{i}}}
\end{aligned}
$$

where $\bar{T}{\widetilde{A_{i}}}=1 / n \sum_{i=1}^{n} T_{\widetilde{A_{i}}}$ is the mean of average quality indices over $i$. In our proposed model we find optimum profit allocation over each interval in such a way that the degree of satisfaction of each agent is very close to 1 . For this purpose, we use a multisection technique in each interval where multiple bisections of the intervals are done in a single iteration. We consider that part of the interval where a better solution is obtained. Again multiple bisections are made 
in the next iteration and a better solution, compared to previous one, is obtained. This process ultimately leads us to the required solution. Here, our target is to obtain such a solution where the optimization is rational and this can be observed from the choice of a particular type of satisfaction function. To clarify this concept we further need to take some definitions as follow.

Definition 3.5 A rational player $i$ satisfies the following conditions.

(i) $S_{\widetilde{s}, v)}^{i}(x)=0$ when $x \leq v\left(\widetilde{s}_{i}\right)$

(ii) $S_{(\widetilde{s}, v)}^{i}(x)=1$ when $x \geq d_{i}$

where $d_{i}=v\left(\widetilde{s}_{i}\right)+e(\widetilde{s}), e(\widetilde{s})=v(\widetilde{s})-\sum_{i=1}^{n} v\left(\widetilde{s}_{i}\right) . d_{i}$ is called the aspiration base (Borkotokey \& Rupok 2012) of the $i$ th player and $e(\widetilde{s})$ is the surplus after making minimum profit allocation to each player. Here, the player is rational who gets at least $v\left(\tilde{s}_{i}\right)$ and then gets the excess amount according to his contribution to the game. Moreover, one question may arise here as to what is our required solution or what is the nature of the required solution which we may consider as the strictly exact allocation. This concept can be clarified by the following definitions.

Definition 3.6 A strictly exact allocation for $\widetilde{s}=\left(c_{1}, c_{2}, \ldots, c_{n}\right) \in \widetilde{F}(Q)$ is an efficient allocation such that $S_{(\widetilde{s}, v)}^{i}\left(x_{1}\right)=S_{(\widetilde{s}, v)}^{i}\left(x_{2}\right)=\ldots=S_{(\widetilde{s}, v)}^{i}\left(x_{n}\right)$ and $S_{(\widetilde{s}, v)}^{i}\left(x_{i}\right) \rightarrow 1$ for $i=1,2, \ldots, n$.

Definition 3.7 Label for $=$ "rational" In an intuitionistic fuzzy coalition $\widetilde{s}=\left(c_{1}, c_{2}, \ldots, c_{n}\right) \in$ $\widetilde{F}(Q)$, an exact allocation $x=\left(x_{1}, x_{2}, \ldots, x_{n}\right)$ is said to be individually strictly rational if $x_{i} \geq$ $v(\widetilde{s})$ and $\frac{\left|x_{i}-x_{j}\right|}{\left|x_{k}-x_{l}\right|} \rightarrow \frac{\left|\mu_{\tilde{A}_{i}}-\mu_{\widetilde{A}_{j}}\right|}{\left|\mu_{\widetilde{A}_{k}}-\mu_{\widetilde{A}_{l}}\right|} \forall i, j, k, l \in N$ and the equality holds for an additive game.

Definition 3.8 An efficient allocation $\hat{x}=\left(\hat{x_{1}}, \hat{x_{2}}, \ldots, \hat{x_{n}}\right)$ is called a better efficient allocation than the efficient allocation $x=\left(x_{1}, x_{2}, \ldots, x_{n}\right)$ if $S_{(\widetilde{s}, v)}^{i}(\hat{x})>S_{(\widetilde{s}, v)}^{i}(x)$.

\section{Exact solution searching protocol}

We attempt to assure at the exact solution in the following manner:

Stage 1. At first we calculate minimum deal index $x(i, \widetilde{s})$ for each agent $i$ and for intuitionistic fuzzy coalition $\widetilde{s}=\left(A_{1}, A_{2}, \ldots, A_{n}\right)$.

Stage 2. Then we calculate interval allocation of profit of each agent $i$ which is of the form $\left[a_{i}^{1}, b_{i}^{1}\right]$ where $a_{i}^{1}$ and $b_{i}^{1}$ are given as

$$
\begin{aligned}
a_{i}^{1} & =x(i, \widetilde{s}) \text { if } T_{\widetilde{A_{i}}} \geq \bar{T}{\widetilde{A_{i}}} \\
& =x(i, \widetilde{s})-x(i, \widetilde{s})\left(\bar{T}{\widetilde{A_{i}}}_{-}-T_{\widetilde{A_{i}}}\right) \text { if } T_{\widetilde{A_{i}}} \leq \bar{T}_{\widetilde{A_{i}}} \\
b_{i}^{1} & =x(i, \widetilde{s}) \text { if } T_{\widetilde{A_{i}}} \leq \bar{T}_{\widetilde{A_{i}}} \\
& =x(i, \widetilde{s})+x(i, \widetilde{s})\left(T_{\widetilde{A_{i}}}-\bar{T}_{\widetilde{A_{i}}}\right) \text { if } T_{\widetilde{A_{i}}} \geq \bar{T}{\widetilde{A_{i}}} .
\end{aligned}
$$

Stage 3. We now use multisection technique on each interval allocation of profit for each agent $i$ and after each iteration we get a better efficient allocation. Suppose at $t$ th iteration we 
get an efficient allocation $x^{t}=\left(x_{1}^{t}, x_{2}^{t}, \ldots, x_{n}^{t}\right)$ and the degree of satisfaction is given by the function

$$
S_{(\widetilde{s}, v)}^{(i, t)}=e^{-\frac{\left|x_{i}^{t}-\bar{x}^{t-1}\right|}{\left|v(\widetilde{s})\left(\mu \widetilde{A_{i}}-\bar{\mu} \widetilde{A_{i}}\right)\right|},}
$$

where $\bar{x}^{t-1}=1 / n \sum_{i=1}^{n} x_{i}^{t-1}$ and length of the interval allocation of profit at $t$ th iteration is $1 / 2^{t}\left(b_{i}^{1}-a_{i}^{1}\right)$. Now, suppose at $(t+1)$ th iteration we get an efficient allocation $x^{t+1}=$ $\left(x_{1}^{t+1}, x_{2}^{t+1}, \ldots, x_{n}^{t+1}\right)$ and the degree of satisfaction is given by the function

$$
S_{(\widetilde{s}, v)}^{(i, t+1)}=e^{-\frac{\left|x_{i}^{t+1}-\bar{x}^{t}\right|}{\left|v(\widetilde{s})\left(\mu \widetilde{A_{i}}-\bar{\mu} \widetilde{A_{i}}\right)\right|},}
$$

where $\bar{x}^{t}=1 / n \sum_{i=1}^{n} x_{i}^{t}$ and length of the interval allocation of profit at $(t+1)$ th iteration is $\frac{1}{2^{t+1}}\left(b_{i}^{1}-a_{i}^{1}\right)$. Now since $x^{t+1}$ is a better efficient allocation compared to $x^{t}$ we have

$$
S_{\widetilde{s}, v)}^{i, t} \leq S_{\widetilde{s}, v)}^{i, t+1}, \quad i=1,2, \ldots, n
$$

We continue with this process until we get at $t=k$ th stage $S_{\widetilde{s}, v)}^{i, k}-S_{\widetilde{s}, v)}^{i, k-1}<\epsilon, i=1,2, \ldots, n$ where $\epsilon$ is the degree of tolerance for the process. Then we say that $\left(x_{1}^{k}, x_{2}^{k}, \ldots, x_{n}^{k}\right)$ is the exact efficient allocation. Here, we need to prove a theorem to show that we actually get better efficient solution at each iteration which is given below

Theorem 4.1 If $x^{t}=\left(x_{1}^{t}, x_{2}^{t}, \ldots, x_{n}^{t}\right)$ be an efficient allocation at the ith iteration and $x^{t+1}=$ $\left(x_{1}^{t+1}, x_{2}^{t+1}, \ldots, x_{n}^{t+1}\right)$ be an efficient allocation at the $(t+1)$ th iteration then $x^{t+1}$ is a better efficient allocation compared to $x^{t}$ i.e., $S_{\widetilde{s}, v)}^{i, t}<S_{\widetilde{s}, v)}^{i, t+1}$ for each agent $i=1,2, \ldots, n$ and intuitionistic fuzzy allocation $\widetilde{s}=\left(A_{1}, A_{2}, \ldots, A_{n}\right)$ where $\widetilde{A_{i}}=\left(\mu_{\widetilde{A_{i}}}, v_{\widetilde{A_{i}}}, \pi_{\widetilde{A_{i}}}\right)$.

Proof. We know from Eqs. (5) and (6) the expressions of $S_{\widetilde{s}, v)}^{(i, t)}$ and $S_{\widetilde{s}, v)}^{(i, t+1)}$. Now, we have,

$$
\begin{aligned}
& \left(x_{i}^{t+1}-x_{i}^{t}\right)<1 / 2^{t}\left(b_{1}^{i}-a_{1}^{i}\right) \text { and }-\left(x_{i}^{t}-x_{i}^{t-1}\right)<1 / 2^{t-1}\left(b_{1}^{i}-a_{1}^{i}\right) \\
\Rightarrow \quad & \left(x_{i}^{t+1}-x_{i}^{t}\right)-\left(x_{i}^{t}-x_{i}^{t-1}\right)<\left(1 / 2^{t}+1 / 2^{t-1}\right)\left(b_{1}^{i}-a_{1}^{i}\right)=3 / 2^{t}\left(b_{1}^{i}-a_{1}^{i}\right)
\end{aligned}
$$

Now when $t \rightarrow \infty, 1 / 2^{t} \rightarrow 0$, and therefore

$$
\begin{aligned}
& \left(x_{i}^{t+1}-x_{i}^{t}\right)<\left(x_{i}^{t}-x_{i}^{t-1}\right)<1 / n \sum_{i=1}^{n}\left(x_{i}^{t}-x_{i}^{t-1}\right)=\bar{x}^{t}-\bar{x}^{t-1} \\
& \Rightarrow\left(x_{i}^{t+1}-\bar{x}^{t}\right)<\left(x_{i}^{t}-\bar{x}^{t-1}\right) \Rightarrow\left|x_{i}^{t+1}-\bar{x}^{t}\right|<\left|x_{i}^{t}-\bar{x}^{t-1}\right| .
\end{aligned}
$$

Hence,

$$
S_{(\widetilde{s}, v)}^{i, t}<S_{(\widetilde{s}, v)}^{i, t+1}
$$

Theorem 4.2 If $S_{\widetilde{s}, v)}^{(i, t)}$ be the degree of satisfaction of an agent $i$ at the th iteration level and $x^{t}=\left(x_{1}^{t}, x_{2}^{t}, \ldots, x_{n}^{t}\right)$ be the efficient allocation at that level then it converges to the strictly exact efficient allocation as $t \rightarrow \infty$ i.e., $S_{\widetilde{s}, v)}^{(i, t)} \rightarrow 1$ as $t \rightarrow \infty$.

Proof. The expression for $S_{\widetilde{s}, v)}^{(i, t)}$ is given in Eq. (5).

Now as $t \rightarrow \infty,\left|x_{i}^{t}-\bar{x}^{t-1}\right| \rightarrow 0$. Hence, $S_{\widetilde{s}, v)}^{(i, t)} \rightarrow 1$ for $i=1,2, \ldots, n$. 
Therefore, we can easily show that

$$
\left|S_{(\widetilde{s}, v)}^{(i, t)}-S_{\widetilde{s}, v)}^{(j, t)}\right|<\epsilon
$$

for $i, j=1,2, \ldots, n$ and $i \neq j$. Here, $\epsilon>0$ is a small number which represents the degree of tolerance. Therefore, the efficient allocation $x^{t}=\left(x_{1}^{t}, x_{2}^{t}, \ldots, x_{n}^{t}\right)$ converges to its strictly exact efficient allocation as $t \rightarrow \infty$. Hence the theorem.

We now need to describe the algorithm of multisection technique by which we find strictly exact efficient allocation. Multisection algorithm (Prasun Kumar Nayak et al 2014) is based on the concept of multiple bisection at single iteration cycle with the comparison of intervals (Prasun Kumar Nayak et al 2014).

\subsection{Algorithm for multisection technique}

Input: $\lambda$ (number of divisions, here 2 ), $l_{i t}$ (lower bound) and $u_{i t}$ (upper bound) of interval allocation of profit for each agent $i$ at iteration $t$.

Output: strictly exact efficient allocation $x_{i}^{\text {opt }}$ for each agent $i$

Step 1://Calculation of step lengths//

for $\mathrm{t}=1 ; \mathrm{t}=\mathrm{t}+1$

calculate step length $h(t)=\left(u_{i t}-l_{i t}\right) / \lambda$

Step 2://Division of concerned region into equal subregions //

Step 2.1: For $i=1$ to $n$

$$
\begin{aligned}
& \text { Calculate } m_{i t}=\left(l_{i t}+u_{i t}\right) / 2 \\
& \text { Calculate } m_{i t 1}=\left(l_{i t}+m_{i t}\right) / 2 \\
& \text { Calculate } m_{i t 2}=\left(m_{i t}+u_{i t}\right) / 2 \\
& \text { Calculate } \bar{m}_{t}=1 / n \sum_{i=1}^{n} m_{i t}
\end{aligned}
$$

Step 2.2: //Call the function $S_{\widetilde{s}, v)}^{(i, t)}(x)$ as defined in Eq. (5)

$$
\begin{aligned}
& \text { Calculate } S_{(\widetilde{s}, v)}^{(i, t)}\left(m_{i t 1}\right) \text { and } S_{\widetilde{s}, v)}^{(i, t)}\left(m_{i t 2}\right) \\
& \quad \text { If } S_{\widetilde{s}, v)}^{(i, t)}\left(m_{i t 1}\right)<S_{\widetilde{s}, v)}^{(i, t)}\left(m_{i t 2}\right) \text { put } l_{i t}=m_{i t} \text { and } u_{i t}=u_{i t},
\end{aligned}
$$

else $l_{i t}=l_{i t}$ and $u_{i t}=m_{i t}$

$E_{t_{i}}=\left[l_{i t}, u_{i t}\right]$ is better efficient allocation interval.

Step 3: //Calculation of difference between consecutive iterations//.

Step 3.1: Calculate difference $w_{t}=S_{\widetilde{s}, v)}^{(i, t)}-S_{(\widetilde{s}, v)}^{(i, t-1)}$ of $E_{t_{i}}$

Step 3.2: If $w_{t}<\varepsilon$

break

else

Step 3.3: Set $E_{i}^{o p t} \leftarrow E_{t_{i}}$

end for

Output

End Multisection 
In the iteration process, it may happen that due to consecutive approximations $(v(\widetilde{s})-$ $\left.\sum_{i=1}^{n} v\left(\tilde{s}_{i}\right)\right)=\delta$, a small positive number exists. We may call it 'Surplus Reward'. This reward should go to that person who delivers highest amount of best quality product. It may again happen that $\left(\sum_{i=1}^{n} v\left(\tilde{s}_{i}\right)-(v)=\delta_{1}\right.$, a small positive number. We may call it 'Unfair Reward' which we may deduct from the reward of that person who delivers least percentage of best quality product. We may make such an adjustment to get efficient allocation at each iterations. Here, we need to remember that if the denominator of the ratio in the power of the satisfaction function is 0 then in the computation process, we put a very small positive number like 0.00001 or 0.000001 instead of 0 to avoid ambiguity. In that case, the iteration method is continued on the basis of closeness of $x_{i}^{t}$ to $\bar{x}^{t-1}$ for each agent $i$.

\section{Numerical example}

One computer manufacturing company established its production unit in a remote place which was far from the city as it got the land there at low cost. It realized then that if it carried different parts from long distance then production cost would increase which would result in increase in cost price of the computers compared to market price. Now the manufacturing company decided to invite other companies manufacturing the required parts to that place where they could get the land at low cost and also they could get better profit in coalition compared to that it would make while conducting their business individually. Let $P_{1}, P_{2}$ and $P_{3}$ be such three companies. $P_{1}$ produces monitors, printers and music system. $P_{2}$ manufactures hard discs, motherboard, RAM and $P_{3}$ manufactures remaining parts of the computers. Now, success of the coalition depends not only on quantity of production but also on the honesty in supply of the products. Every partner invests the same amount of units but if a partner tries to get more profit by providing low quality products then the total profit decreases and he has to take responsibility for the convenience. So, we take an intuitionistic fuzzy number for each agent to make the quality assessment which are nothing but percentage of products of specific quality. Suppose, these intuitionistic fuzzy numbers for $P_{1}, P_{2}$ and $P_{3}$ are respectively $\left(\mu_{1}, v_{1}, \pi_{1}\right)=(0.5,0.3,0.2),\left(\mu_{2}, \nu_{2}, \pi_{2}\right)=(0.6,0.1,0.3)$ and $\left(\mu_{3}, \nu_{3}, \pi_{3}\right)=(0.7,0.2,0.1)$. Let $v\left(\widetilde{s}_{1}\right)=8, v\left(\widetilde{s}_{2}\right)=12$ and $v\left(\widetilde{s}_{1}\right)=15, v(\widetilde{s})=45$. Then we get the interval allocations of profit as [9.437684, 10.777778], [14.278660, 15.333333] and [18.888889, 22.536743]. Now if we apply multisection technique then the result obtained can be written as follow.

\begin{tabular}{lll}
\hline Iterations & \multicolumn{1}{c}{$x^{t}=\left(x_{1}^{t}, x_{2}^{t}, x_{3}^{t}\right)$} & $S_{(\widetilde{s}, v)}^{(i, t)}$ \\
\hline$t=1$ & $(9.481188,14.805996,20.712816)$ & $(0.989618,0.999965,0.989694)$ \\
$t=2$ & $(10.129482,15.069664,19.800854)$ & $(0.990506,0.999965,0.990178)$ \\
$t=2$ & $(10.45363,15.201498,19.344872)$ & $(0.990854,0.999965,0.990516)$ \\
$t=4$ & $(10.694021,15.135581,19.170398)$ & $(0.991028,0.999965,0.990685)$ \\
$t=5$ & $(10.735899,15.102623,19.161478)$ & $(0.991115,0.999965,0.990770)$ \\
$t=6$ & $(10.756838,15.086143,19.157019)$ & $(0.991158,0.999965,0.990812)$ \\
$t=7$ & $(10.767307,15.077904,19.154789)$ & $(0.991180,0.999965,0.990834)$ \\
$t=8$ & $(10.772543,15.073784,19.153673)$ & $(0.991191,0.999965,0.990844)$ \\
$\mathbf{t}=\mathbf{9}$ & $(\mathbf{1 0 . 7 7 5 1 6 0 , 1 5 . 0 7 1 7 2 4 , 1 9 . 1 5 3 1 1 6 )}$ & $(\mathbf{0 . 9 9 1 1 9 6 , 0 . 9 9 9 9 6 5 , 0 . 9 9 0 8 4 9 )}$ \\
$t=10$ & $(10.776468,15.070694,19.152838)$ & $(0.991199,0.999965,0.990852)$ \\
$t=11$ & $(10.777122,15.070179,19.152699)$ & $(0.991200,0.999965,0.990853)$ \\
\hline
\end{tabular}


The degree of tolerance has been considered as 0.00001 and the optimum result is obtained at 9 th iteration.

\subsection{Results and discussions}

In the given example, we see that the strictly exact efficient allocation for the intuitionistic fuzzy coalition converges at 9th iteration with degree of tolerance 0.00001 and it is $x^{t}=$ $(10.775160,15.071724$, and 19.153116). We now see that if we compare our result with those of existing techniques then it is more rational. For example, if we compare our result with (Borkotokey \& Rupok 2012) then we get the following differences.

(i) In our example, $\frac{\left|\mu_{\widetilde{A}_{1}}-\mu_{\widetilde{A}_{2}}\right|}{\left|\mu_{\widetilde{A}_{3}}-\mu_{\widetilde{A}_{2}}\right|}=0.1 / 0.1=1$ and

$$
\begin{array}{r}
\frac{\left|x_{1}^{t}-x_{2}^{t}\right|}{\left|x_{3}^{t}-x_{2}^{t}\right|}=\frac{|10.775160-15.071724|}{|19.153116-15.071724|}=4.296564 / 4.081392 \simeq 1.05 \\
\text { Therefore, }\left|\frac{\left|x_{1}^{t}-x_{2}^{t}\right|}{\left|x_{3}^{t}-x_{2}^{t}\right|}-\frac{\left|\mu_{\widetilde{A}_{1}}-\mu_{\widetilde{A}_{2}}\right|}{\left|\mu_{\widetilde{A}_{3}}-\mu_{\widetilde{A}_{2}}\right|}\right|=0.05 .
\end{array}
$$

But this result in Borkotokey \& Rupok (2012) is obtained as $x^{t}=(12.2174,16.0664$, 16.7162) and $\frac{\left|\mu_{\widetilde{A}_{1}}-\mu_{\widetilde{A}_{2}}\right|}{\left|\mu_{\widetilde{A}_{3}}-\mu_{\widetilde{A}_{2}}\right|}=0.1 / 0.1=1$ and

$$
\begin{aligned}
\frac{\left|x_{1}^{t}-x_{2}^{t}\right|}{\left|x_{3}^{t}-x_{2}^{t}\right|}= & \frac{|12.2174-16.0664|}{|16.7162-16.0664|}=3.849 / 0.6498 \simeq 5.92 \\
& \text { Therefore, }\left|\frac{\left|x_{1}^{t}-x_{2}^{t}\right|}{\left|x_{3}^{t}-x_{2}^{t}\right|}-\frac{\left|\mu_{\widetilde{A}_{1}}-\mu_{\widetilde{A}_{2}}\right|}{\left|\mu_{\widetilde{A}_{3}}-\mu_{\widetilde{A}_{2}}\right|}\right|=4.92 .
\end{aligned}
$$

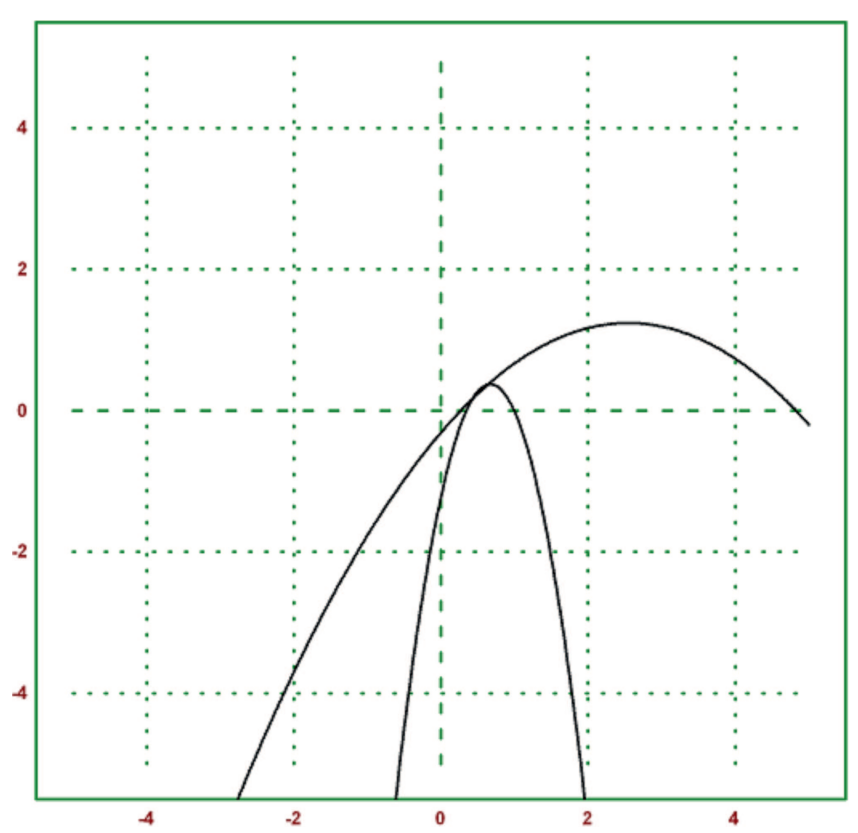

Figure 2. Comparison of results w.r.t (Borkotokey \& Rupok 2012). 
If we compare Eqs. (7) and (8) we see that our result is more rational in accordance with Definition (3.7) i.e., the profit allocation as compared to supply of best quality of products is more rational compared to (Borkotokey \& Rupok 2012).

Figure 2 represents a comparison of our result with the result corresponding to that in Borkotokey \& Rupok (2012). Here, the curve with lesser radius of curvature represents our result and other curve represents that in Borkotokey \& Rupok (2012). It indicates that the result corresponding to our approach is more rational compared to that in Borkotokey \& Rupok (2012).

(ii) In our approach the rate of convergence is more as compared to Borkotokey \& Rupok (2012) as the number of iterations is lesser in our technique.

(iii) In our model, choice of satisfaction function has been made in such a way that degree of satisfaction of each agent $i$ is very high and very close to 1 .

\section{Conclusion}

In this paper, a dynamic solution method has been proposed where a rational profit allocation has been made among rational players in a joint venture project such that their degree of satisfaction approaches to its highest level. The coalition is considered in a fuzzy environment modelled by an intuitionistic number to judge their quality of participation. The corresponding profit allocation method actually inspires them for betterment of their performance to get better reward. Here, our approach has successive iterations where the profit allocation of each agent $i$ at a particular iteration converges to the arithmetic mean of previous iteration over $i$. Here, we can also think the situation differently with weighted arithmetic mean. Another possible extension of the methodology is the formulation of the technique for large games. The concept can also be considered in multi-dimensional space.

\section{References}

Aubin J P 1982 Mathematical methods of game and economic theory (rev. edn.). North-Holland, Amsterdam 7: 1-619

Azrieli Y and Lehrer E 2007 On some families of cooperative fuzzy games. Int. J. Game Theory 36: 1-15

Borkotokey S 2008 Modelling a solution concept to cooperative games with fuzzy coalitions through negotiation via mediator. Math Forum Spl. 21: 33-40

Borkotokey S and Rupok Neog 2012 Allocating profit among rational players in a fuzzy coalition: A game theoretic model. Group Decis. Negot. 21: 439-459

Branzei R, Dimitrov D and Tijs S 2004 Hypercubes and compromise values for cooperative fuzzy games. Eur. J. Oper. Res. 155: 733-740

Branzei R, Dimitrov D and Tijs S 2005 Models in cooperative game theory: crisp, fuzzy and multichoice games. Lecture Notes In Economics and Mathematical Systems. Berlin, Springer, 556

Butnariu D 1980 Stability and shapley value for an n-persons fuzzy game. Fuzzy Sets Syst. 4: 63-72

Dieckmann T 2002 Dynamic coalition formation and the core. J. Econ. Behav. Organ 49: 363-380

Mares M and Vlach M 2001 Linear coalition games and their fuzzy extensions. Int. J. Uncertain Fuzziness Knowledge Based Syst. 9: 341-354

Mares M and Vlach M 2006 Fuzzy coalitional structures. Mathware Soft Computation 13: 59-70

Moore R E 1979 Method and application of interval analysis. SIAM, Philadelphia

Prasun Kumar Nayak, Sibasis Bandyopadhyay and Madhumangal Pal 2014 An algorithm for solution of interval games. Int. J. Oper. Res. 20(2): 207-225

Ray D and Vohra R 1997 Equilibrium binding agreements. J. Econ. Theory 73: 30-78 
Ray D and Vohra R 1999 A theory of endogenous coalition structure. Games Econ. Behav. 26: 286-336

Ray D and Vohra R 2001 Coalitional power and public goods. J. Pol. Econ. 109: 1355-1384

Romero Cortes J C and Sheremotov L B 2002 Model of cooperation in multi-agent systems with fuzzy coalitions. CEEMAS, LNAI 2296: 263-272

Sakawa M 1983 Interactive computer programme for fuzzy linear programming with multiple objectives. Int. J. Man-Mach. Stud. 18: 489-503

Seikh M R, Nayak Prasun and Pal M 2013 Notes on triangular intuitionistic fuzzy number. Int. J. Math. Oper. Res. 5(4): 446-465

Shapley L S 1953 A value for n-person games. In: Kuhn H W, Tucker, A W (Eds.), Contributions to the theory of Games II. In: Ann. Math. Stud. 2: 307-317

Xiaodong Liu, Jiuqiang Liu and Cui Li 2013 Average monotonic cooperative fuzzy games. Fuzzy Sets Syst. 231: 95-107 